\title{
28 Research Soure \\ The first case of feline cytauxzoonosis in Germany: clinical description and molecular confirmation
}

Luciana Catalina Panait ( $\square$ luciana.rus@usamvcluj.ro)

University of Agricultural Sciences and Veterinary Medicine of Cluj-Napoca https://orcid.org/00000003-4360-5790

\section{Graham Stock}

IDEXX Laboratories, D-71636 Ludwigsburg.

\section{Majda Globokar}

IDEXX Laboratories, D-71636 Ludwigsburg

Jörg Balzer

IDEXX Laboratories, D-71636 Ludwigsburg

\section{Bernhard Groth}

Vet Practice, D-66740 Saarlouis

Andrei Daniel Mihalca

University of Agricultural Sciences and Veterinary Medicine of Cluj-Napoca

Nikola Pantchev

IDEXX Laboratories, D-71636 Ludwigsburg

\section{Research}

Keywords: Cytauxzoon sp., domestic cat, piroplasm, vector-borne disease, FIV, Felis silvestris, Germany, PCR

Posted Date: January 16th, 2020

DOI: https://doi.org/10.21203/rs.2.21005/v1

License: (c) (i) This work is licensed under a Creative Commons Attribution 4.0 International License.

Read Full License 


\section{Abstract}

\section{Background}

Cytauxzoonosis is gaining clinical importance in recent years, being described as an emerging tick-borne disease of domestic and wild felids caused by protozoans of the genus Cytauxzoon. While in the Americas the condition is described as a fatal disease, in Europe, reports on the clinical expression of the infection are scarce. This study describes the first case of Cytauxzoon sp. infection in a domestic cat in Germany.

Methods

Clinical and laboratory findings, and molecular and DNA sequencing results were collected from a domestic cat suspected for cytauxzoonosis after the microscopic examination of a blood smear.

Results

A 6-year-old male domestic cat living in Saarlouis (Germany) was presented with a history of recent anorexia and weight loss. The cat had an outdoor lifestyle and had not travelled abroad. Serum clinical chemistry analysis revealed azotaemia with markedly increased symmetric dimethylarginine, hypercreatinemia, hyperphosphatemia and hypoalbuminemia. Moreover, a mild non-regenerative anaemia was present. Approximately one year prior these findings, the patient was diagnosed with a FIV infection. These results point toward a decreased glomerular filtration rate, presumably as a result of a kidney dysfunction. Therefore, a glomerulonephropathy was suspected. Round to oval signet-ring shaped intraerythrocytic organisms, morphologically suggestive for a piroplasm, were revealed during blood smear evaluation with a degree of parasitaemia of $32.95 \%$. PCR analyses and sequencing of the partial $18 \mathrm{~S}$ gene confirmed the presence of a Cytauxzoon sp. infection, with $99-100 \%$ nucleotide sequence identity with previously published Cytauxzoon sp. isolates.

\section{Conclusion}

This study provides the first case of molecularly confirmed Cytauxzoon sp. infection in a domestic cat in Germany and describes the clinical picture of the disease in association with FIV infection. These findings suggest that cytauxzoonosis should be considered as a differential diagnosis in cases of anaemia in domestic outdoor cats, particularly in areas where wild felid populations are present.

\section{Background}

A tick-borne and Babesia-like pathogen in felids, Cytauxzoon felis, is gaining clinical importance in recent years, although its occurrence outside the Americas is not reported. Domestic cats infected with $\mathrm{C}$. felis generally develop a peracute and highly fatal disease [1]. 
Furthermore, other closely related piroplasms infecting domestic and wild felids have been reported. Cytauxzoon manul was described in naturally infected Pallas's cats (Otocolobus manul) from Mongolia $[2,3]$. In Europe, an unnamed species of Cytauxzoon was reported in domestic cats from Spain $[4,5]$, France [6, 7], Italy [8, 9], Portugal [10], and Switzerland [11], but also from wild felids in Spain [12-18], Romania [19], Italy [20], and Bosnia and Herzegovina [21]. Studies on Cytauxzoon sp. in Europe show different infection rates depending on countries and species involved. While the infection rate in domestic cats is ranging from $0.8 \%$ in France [6] to $23 \%$ in Italy [8], in wild felids the assessed prevalence is generally higher than 50\% in Romania [19], Bosnia and Herzegovina [21], and Spain [12, 14].

The European isolates of Cytauxzoon seem to be less virulent than C. felis and the clinical manifestations appear to be associated with immune-mediated diseases or secondary infections $[5,11]$. Due to non-clinical carrier state as well as non-specific clinical signs like lethargy, anorexia, anaemia, fever, weight loss, tachycardia, tachypnoea, diarrhoea, and vomiting, clinical reports for Cytauxzoon sp. infections in Europe are scarce [7-10].

The present paper reports the first molecular confirmation of Cytauxzoon sp. infection in Germany and provides a detailed clinical picture of the disease in association with feline immunodeficiency virus (FIV) infection.

\section{Methods}

\subsection{Patient}

In March 2017, a 6-year-old male domestic cat was referred to a veterinary facility in Saarlouis $\left(49.31^{\circ} \mathrm{N}\right.$, $6.75^{\circ} \mathrm{E}$ ), a small city located in the south-west part of Germany, with unspecific clinical signs (detailed case description is provided in the results section). The cat had an outdoor lifestyle and had never travelled abroad.

\subsection{Clinical blood analyses}

In March 2017, a complete blood count and serum clinical biochemical analysis (including the assessment of liver, kidney, and pancreas function) were performed at IDEXX reference laboratory in Ludwigsburg, Germany (Table 1). Symmetric dimethylarginine (SDMA) was measured using a commercially available high-throughput immunoassay (IDEXX SDMA Test, IDEXX Laboratories Inc.) [22]. No urine examination was performed. 
Table 1

Clinico-pathological values from March 2017, compared to respective values from January 2016; for clinical chemistry, only abnormal values were included

\begin{tabular}{|c|c|c|c|}
\hline Parameter & $\begin{array}{l}\text { Measured value (unit) in } \\
2016\end{array}$ & $\begin{array}{l}\text { Measured value (unit) in } \\
2017\end{array}$ & Reference interval (unit) \\
\hline Urea nitrogen & $26 \mathrm{mg} / \mathrm{dl}$ & $69 \mathrm{mg} / \mathrm{dl} *$ & $16-38(\mathrm{mg} / \mathrm{dl})$ \\
\hline Creatinine & $0.9 \mathrm{mg} / \mathrm{dl}$ & $2.8 \mathrm{mg} / \mathrm{dl}{ }^{\star}$ & $<1.9(\mathrm{mg} / \mathrm{dl})$ \\
\hline Phosphate & $1.5 \mathrm{mmol} / \mathrm{l}$ & $2.4 \mathrm{mmol} / \mathrm{l}^{\star}$ & $0.8-2.2(\mathrm{mmol} / \mathrm{l})$ \\
\hline SDMA & $4 \mu \mathrm{g} / \mathrm{dl}$ & $45 \mu \mathrm{g} / \mathrm{dl} *$ & $<=14(\mu \mathrm{g} / \mathrm{dl})$ \\
\hline Albumin & $4.4 \mathrm{~g} / \mathrm{dl}$ & $1.8 \mathrm{~g} / \mathrm{dl} *$ & $2.7-4.4(\mathrm{~g} / \mathrm{dl})$ \\
\hline $\begin{array}{l}\text { Albumin-globulin } \\
\text { ratio }\end{array}$ & 1.52 & $0.35^{\star}$ & $>0.57$ \\
\hline $\begin{array}{l}\text { Alkaline } \\
\text { phosphatase }\end{array}$ & $77 \mathrm{U} / \mathrm{I}^{\star}$ & $162 \mathrm{U} / \mathrm{I}^{\star}$ & $<73(\mathrm{U} / \mathrm{I})$ \\
\hline Erythrocytes & $10.1 \mathrm{~T} / \mathrm{I}$ & $6.5 \mathrm{~T} /{ }^{*}$ & $7.1-11.5(\mathrm{~T} / \mathrm{I})$ \\
\hline Haemoglobin & $13.4 \mathrm{~g} / \mathrm{dl}$ & $8.9 \mathrm{~g} / \mathrm{dl} *$ & $10.3-16.2(\mathrm{~g} / \mathrm{dl})$ \\
\hline Haematocrit & 43 & 29.2 & $28.2-52.7 \%$ \\
\hline MCV & 43 & 44.9 & $39-56$ (fl) \\
\hline $\mathrm{MCH}$ & 13 & 13.7 & $12.6-16.5(\mathrm{pg} / \mathrm{cell})$ \\
\hline $\mathrm{MCHC}$ & 31 & 30.6 & $28.5-37.8(\mathrm{~g} / \mathrm{dl})$ \\
\hline Thrombocytes & 275 & 186 & $155-641(\mathrm{G} / \mathrm{l})$ \\
\hline Reticulocytes & $37000 / \mu 1^{*}$ & $15000 /\left.\mu\right|^{*}$ & $\begin{array}{l}>=50000 / \mu l \text { (when } \\
\text { anaemic) }\end{array}$ \\
\hline Leukocytes & 8.2 & 18.2 & $3.9-19(\mathrm{G} / \mathrm{I})$ \\
\hline Neutrophils & $6231 / \mu \mathrm{l}$ & $16781 /\left.\mu\right|^{*}$ & $2620-15170 / \mu \mathrm{l}$ \\
\hline Lymphocytes & 1575 & 912 & $850-5850 / \mu \mathrm{l}$ \\
\hline Monocytes & $320 / \mu \mathrm{l}$ & $547 / \mu 1^{*}$ & $40-530 / \mu \mathrm{l}$ \\
\hline \multicolumn{4}{|c|}{ *Values that fall outside the reference range } \\
\hline Additional file 1 & & & \\
\hline
\end{tabular}

Approximately one year prior to these investigations (January 2016), a blood sample from the same patient was sent to IDEXX laboratories for similar hematologic and clinical biochemical analysis 
(Table 1). At that time, the blood sample was also tested for feline leukaemia virus (FeLV) antigen (p27; PetChek FeLV, IDEXX Laboratories Inc.), as well as for antibodies against FIV (p24/gp40; PetChek Plus Anti-FIV, IDEXX Laboratories Inc) and feline coronavirus (FCoV) (FCoV ELISA cat, Afosa Germany).

\subsection{Microscopic examination of blood smears}

May Grünwald-Giemsa-stained blood smears were prepared from peripheral blood and were evaluated for the presence of intraerythrocytic piroplasms. The degree of parasitaemia was estimated by counting the number of piroplasm-infected erythrocytes per 2000 erythrocytes with an Olympus BX61 microscope at $\times$ 1,000 magnification. The measuring of the well-defined organisms (with a characteristic shape, $n=100$ ) was assessed with a DP72 camera and Cell^F software (Olympus Corporation, Japan).

\subsection{Molecular analyses and DNA sequencing}

Total nucleic acid was extracted using the QIAamp DNA Blood BioRobot MDx kit (Qiagen, Hilden, Germany) following the manufacturer's instructions. A polymerase chain reaction (PCR) assay for Piroplasmida targeting the 18S rRNA gene was performed according to Carret et al. 1999 [23] and Katargina et al. 2011 [24]. Furthermore, a nested and a conventional PCR protocol were used for the amplification of the partial 18S rRNA gene of the phylum Apicomplexa, with the primer pairs BTH1F/BTH-1R, GF2/GR2 and 7549/7548, respectively, following previously published reaction procedures and protocols $[3,25]$. PCR products were visualised by electrophoresis in a $2 \%$ agarose gel. After purification, the amplicons were submitted for sequencing on both strands (at Macrogen, Netherlands). Consensus sequences were compared to the sequences available in GenBank ${ }^{\text {TM }}$ dataset by Basic Local Alignment Search Tool (BLAST) analysis.

\section{Results}

\subsection{Case description}

The cat was presented to the veterinary clinic in March 2017 with a history of recent anorexia. According to the owner, the patient had refused to eat in the last 3 days and lost approximately $1.5 \mathrm{~kg}$ in the previous few weeks. Episodes of vomiting could not be excluded due to the outdoor lifestyle of the cat. The patient received symptomatic therapy for 5 days with maropitant citrate (Cerenia ${ }^{\circledR}, 1 \mathrm{mg} / \mathrm{kg}, \mathrm{SC}$ ), metamizole (Novalgin ${ }^{\circledR} ; 35 \mathrm{mg} / \mathrm{kg}$, IM), amoxicillin (Betamox ${ }^{\circledR}$ long acting $150 \mathrm{mg} / \mathrm{ml}, 15 \mathrm{mg} / \mathrm{kg}, \mathrm{SC}$ ) and fluids (Lactated Ringer's solution, $15 \mathrm{ml} / \mathrm{kg}, \mathrm{SC}$ ). Despite the therapy, the cat was euthanized on the 5th day, due to the worsening of the clinical state, being presented in a lateral decubitus with severe lethargy and ataxia. Nevertheless, the owner elected against necropsy. No information regarding the history of tick infestation or previous antiparasitic treatments were available.

\subsection{Haematology and blood biochemistry}

In March 2017, the haematological analyses revealed azotaemia with markedly increased SDMA, hypercreatinemia, as well as hyperphosphatemia and hypoalbuminemia. Furthermore, a mild non- 
regenerative anaemia was present. Abnormal laboratory findings are shown in Table 1. The results indicated decreased glomerular filtration rate, likely due to kidney disease. Urinalysis was not performed to assess specific gravity, protein concentration or sediment.

In January 2016, the hematological and clinical chemistry variables had been within normal limits. FeLV and FCoV tests were negative, but a positive FIV antibody test was obtained.

\subsection{Blood smear microscopy}

Blood smear examination in March 2017 revealed morphologically unremarkable leukocytes, while erythrocytes displayed no significant anisocytosis or polychromasia. Blood smear evaluation revealed round to oval signet-ring shaped organisms inside the erythrocytes, with a lightly basophilic cytoplasm and a basophilic nucleus usually located in an eccentric position (Additional file 1: Fig. 1).

One to four merozoites were observed within individual red blood cells. The mean length of the welldefined forms was $1.2 \pm 0.2 \mu \mathrm{m}$ and the measured width was $1.0 \pm 0.2 \mu \mathrm{m}$. The intensity of parasitaemia was estimated at $32.95 \%$. The intracellular parasites were morphologically suggestive for a piroplasm. Moreover, the typical signet ring-shaped organisms could be clearly differentiated from feline haemotropic mycoplasma due to their evident large nuclear area (see also [1]).

Microscopic examination of the blood smear in January 2016 revealed no blood cell morphologic abnormalities and no microorganisms.

\subsection{Molecular detection and characterization}

The presence of Cytauxzoon sp. DNA was confirmed by positive PCR results in all three protocols and subsequent sequencing. The longest consensus sequence available (1010 bp) was submitted to GenBank $^{\text {TM }}$ under the accession number MN629916. BLAST analysis of the partial 18S gene revealed 99.9\% nucleotide sequence identity with a GenBank ${ }^{\text {TM }}$ sequence of Cytauxzoon sp. originating from a domestic cat from France (accession number EU622908.1). Moreover, similarities of $100 \%$ were identified with sequences of Cytauxzoon sp. (MF503141, MF503143-6, KU306941-3, KU306945-8; KX881967) obtained from pet cats from Switzerland and France with clinical cytauxzoonosis.

\section{Discussion}

The study reported herein describes the clinical picture, laboratory findings and diagnostic procedures of a Cytauxzoon sp. clinical infection in a domestic cat from Germany. A previous piroplasmid fatal infection was described in a captive Bengal tiger from a zoo in Germany, when parasitic inclusions resembling Cytauxzoon spp. were visualised in histological sections of various tissues, the largest number being found in blood vessels of lymph nodes and spleen. Even though there was no evidence of tick infestation in the tiger, three bobcats originating from an American zoo were incriminated as a possible source of the infection [26]. 
The cat patient in the current report was also diagnosed with a FIV infection, being presented in a critical condition a few days following clinical examination due to suspected kidney disease. Since in dogs, clinical cases of babesiosis and theilerioses are known to cause acute or chronic nephropathy or glomerulonephritis $[27,28]$, the renal disturbances observed in this patient could have been attributed to the piroplasmid infection, to FIV or both. Previous studies have confirmed that FIV infection can induce the accumulation of immune complexes in renal tissue, therefore, a causative relationship between FIV infection and glomerulonephritis has been posited [29-32]. In the light of this information and the laboratory results (hypoalbuminemia pointing toward a renal loss of protein, increased creatinine and phosphate values as well as a very high SDMA value) one could postulate that the patient had glomerular disease due to immune-complex deposition in terms of a membranoproliferative glomerulonephritis. Other potential causes of hypalbuminaemia in this patient would include decreased production, other causes of loss (i.e. blood loss, protein-losing enteropathy) or haemodilution. In cases of renal injury, SDMA serum concentration increases earlier than creatinine, remaining elevated also in cases of chronic kidney disease (on average with $40 \%$ reduction of glomerular filtration rate, compared with up to $75 \%$ reduction needed to increase the creatinine value) [33].

Regarding the pathogenesis of FIV infection, cats remain asymptomatic for several years until functional immunodeficiency (by increasing the susceptibility to secondary infections and neoplasia) and/or immune-mediated diseases will eventually translate into clinical manifestation around 4 to 6 years of age or older [31]. In the present case, the cat did not display any noticeable symptoms at 5 years of age, approximately 1 year before merozoites resembling Cytauxzoon sp. were detected in high numbers in the blood smear. Therefore, it can be presumed that, as a result of immunosuppression caused by FIV, the piroplasm contributed as an opportunistic factor triggering the clinical picture. Furthermore, several reports have identified a positive relationship between FIV infection or other immune-mediated conditions and cytauxzoonosis in Europe $[5,11]$. The clinical role of Cytauxzoon sp. in domestic cats without immunosuppression and coinfections remains debatable, as the information about pathogenesis and clinical involvement is limited. Although some cases of clinical illness and fatal outcome have been described $[7,10,11]$, the majority of Cytauxzoon sp. infected cats were apparently healthy, with only few animals showing mild anaemia syndrome $[5,8,9,11]$.

As the molecular confirmation of Cytauxzoon sp. infection was established post-mortem, no specific antipiroplasm treatment was applied. Although, in C. felis infection, the recommended therapeutic protocol consists of atovaquone and azithromycin [34], the optimal therapy for Cytauxzoon sp. infection remains unknown due to the lack of controlled clinical studies. In a recently published case report, three 2 month old kittens infected with Cytauxzoon sp. were treated with a combination of atovaquone and azithromycin, with a putative success [11]. Also, previously published Cytauxzoon sp. infections in domestic cats have been medicated with various antiprotozoal drugs, including imidocarb dipropionate and doxycycline [7-10]. Clinical studies in Europe reported that a single imidocarb dipropionate administration was not successful in treating cytauxzoonosis, although the addition of doxycycline cleared parasitaemia in one cat $[7,9]$. 
Based on the sequencing results performed on the partial 18S rRNA gene amplification product, Cytauxzoon sp. detected in the present case revealed a high homology (99-100\%) with Cytauxzoon sp. reported in European domestic felids $[7,11]$ and with Cytauxzoon manul $(99.80 \%)$ described from Pallas' cats from Mongolia [3]. In addition, the sequence displayed a similarity of $95 \%$ to C. felis $18 \mathrm{~S}$ rRNA partial sequences deposited in GenBank®.

Microscopic examination of the blood smear revealed a degree of parasitaemia of approximately $33 \%$. Other clinical studies showed lower levels of parasitaemia [ 7-9, 15], while similar percentages of piroplasm-infected erythrocytes were observed in the blood smear of three cats with cytauxzoonosis from Switzerland [11].

A significant association between the detection of Cytauxzoon sp. DNA in European domestic cats and outdoor lifestyle has been found, particularly in rural areas [5, 8]. In agreement with previous results, the cat from the present study showed an outdoor lifestyle. This would suggest a higher risk of infection, potentially due to exposure to tick vectors and wildlife reservoirs. For $\mathrm{C}$. felis infections of domestic cats, the American bobcat (Lynx rufus) is assumed to act as the main reservoir [35] and Amblyomma americanum and Dermacentor variabilis are the confirmed tick-vectors $[36,37]$. However, for the European isolates of Cytauxzoon sp. little is known about vectors and routes of transmission. In Europe, high prevalences of Cytauxzoon sp. infection were found in Felis silvestris in Romania [19], Italy [20], Bosnia and Herzegovina [21], and Spain [12, 14]. Moreover, Germany has one of the biggest European populations of Felis silvestris, that is concentrated in the low altitude mountain areas [38]. Studies on wild cat distribution suggest that Saarlouis city is surrounded by an abundant population [39]. Consequently, considering that they might live in close proximity to urban and rural areas and crossbreed with domestic cats, it is possible that wild cats play a central role in the transmission of Cytauxzoon sp. in west Germany, as in probably all of Europe. Ixodes ricinus is the most commonly found tick in Germany [40], and it has already been hypothesized to be a possible vector for Cytauxzoon sp. [19].

This study describes, to the best of our knowledge, the first case of molecularly confirmed Cytauxzoon sp. infection in Germany.

\section{Conclusions}

This case provides a new geographical record of Cytauxzoon sp. infection in domestic cats in Central Europe and describes the clinical picture of this disease in association with FIV infection. These findings advocate that cytauxzoonosis should be considered as a differential diagnosis in cases of anaemia in domestic cats with an outdoor lifestyle, particularly in areas where populations of wild felids are present. Additional studies are required to identify the possible arthropod vectors which may be involved in the transmission, and to clarify the relationship between Cytauxzoon sp. infection in cats and the presented clinical signs.

\section{Declarations}




\section{Ethics approval and consent to participate}

All clinical procedures were done as part of the diagnostic workup and treatment of the cat.

\section{Consent for publication}

Not applicable.

\section{Availability of data and material}

The datasets supporting the conclusions of this article are included within the article.

\section{Competing interests}

The authors declare that they have no competing interests.

\section{Funding}

Part of the work was supported by the UEFISCDI Grant Agency Romania, grant number PCCDI 57/2018 and by the University of Agricultural Sciences and Veterinary Medicine of Cluj-Napoca, Romania.

\section{Authors' contributions}

LCP wrote the manuscript and performed the microscopical analyses, molecular assays and sequence analyses of the sample. GS, MG, JG and NP were responsible for the serum/blood analyses, microscopical examination of the blood smear and the initial molecular diagnosis. BG performed anamnesis, clinical examination and treatment. ADM and NP supervised and critically revised the manuscript for important intellectual content. NP drafted the original manuscript. All authors read and approved the final manuscript.

\section{Acknowledgements}

Part of this research has been supported from PCCDI57/2018.

\section{References}

1. Cohn LA, Birkenheuer AJ. Cytauxzoonosis. In: Sykes L, Greene C, editors. Infectious diseases of the dog and cat. 4th Edition. St. Louis: Elsevier Saunders; 2012. p. 764-71.

2. Ketz-Riley CJ, Reichard MV, Van Den Bussche RA, Hoover JP, Meinkoth J, Kocan AA. An intraerythrocytic small piroplasm in wild-caught Pallas's cats (Otocolobus manul) from Mongolia. Wildl. Dis. 2003;39:424-30.

3. Reichard MV, Van Den Bussche RA, Meinkoth JH, Hoover JP, Kocan AA. A new species of Cytauxzoonfrom Pallas' cat caught in Mongolia and comments on the systematic and taxonomy of piroplasmids. Parasitol. 2005;91:420-7. 
4. Criado-Fornelio A, Gónzalez-del-Rıo MA, Buling-Saraña A, Barba-Carretero JC, 2004. The "expanding universe" of piroplasms. Vet. Parasitol. 2004;119:337-45.

5. Díaz-Regañón D, Villaescusa A, Ayllón T, Rodríguez-Franco F, Baneth G, Calleja-Bueno L, et al. Molecular detection of Hepatozoon and Cytauxzoon sp. in domestic and stray cats from Madrid, Spain. Parasit. Vectors. 2017;10:112.

6. Criado-Fornelio A, Buling A, Pingret JL, Etievant M, Boucraut-Baralon C, Alongi A, et al.. Hemoprotozoa of domestic animals in France: prevalence and molecular characterization. Parasitol. 2009;159:73-6.

7. Legroux JP, Halos L, René-Martellet M, Servonnet M, Pingret JL, Bourdoiseau G. et al. First clinical case report of Cytauxzoon infection in a domestic cat in France. BMC Vet. Res. 2017;13:81.

8. Carli E, Trotta M, Chinelli R, Drigo M, Sinigoi L, Tosolini P, et al. Cytauxzoon infection in the first endemic focus described in domestic cats in Europe. Vet. Parasitol. 2012;183:343-52.

9. Carli E, Trotta M, Bianchi E, Furlanello T, Caldin M, Pietrobelli M, et al. Cytauxzoon infection in two free ranging young cats: clinicopathological findings, therapy and follow up. Turkiye Parazitol. Derg. 2014;38:185-9.

10. Alho AM, Silva J, Fonseca MJ, Santos F, Nunes C, de Carvalho LM, et al. First report of Cytauxzoon sp. infection in a domestic cat from Portugal. Vectors. 2016;9:220.

11. Nentwig A, Meli ML, Schrack J, Reichler IM, Riond B, Gloor C, et al. First report of Cytauxzoon infection in domestic cats in Switzerland: natural and transfusion-transmitted infections. Parasit. Vectors. 2018;11:292.

12. Barandika JF, Espí A, Oporto B, Del Cerro A, Barral M, Povedano I, et al. Occurrence and genetic diversity of piroplasms and other apicomplexa in wild carnivores. Parasitol. Open. 2016;2:e6.

13. García-Bocanegra I, Dubey JP, Martínez F, Vargas A, Cabezón O, Zorrilla I, et al. Factors affecting seroprevalence of Toxoplasma gondii in the endangered Iberian lynx (Lynx pardinus). Vet. Parasitol. 2010;167:36-42.

14. León Cl, García-Bocanegra I, McCain E, Rodríguez E, Zorrilla I, Gómez AM, et al. Prevalence of selected pathogens in small carnivores in reintroduction areas of the lberian lynx (Lynx pardinus). Rec. 2017;180;252-5.

15. Luaces I, Aguirre E, García-Montijano M, Velarde J, Tesouro MA, Sánchez C, et al. First report of an intraerythrocytic small piroplasm in wild Iberian lynx (Lynx pardinus). Wildl. Dis. 2005;41:810-5.

16. Meli ML, Cattori V, Martínez F, López G, Vargas A, Simón MA, et al. Feline leukemia virus and other pathogens as important threats to the survival of the critically endangered Iberian lynx ( $L y n x$ pardinus). PLoS One. 2009;4:e4744.

17. Millán J, Naranjo V, Rodríguez A, De La Lastra JP, Mangold AJ, De La Fuente J. Prevalence of infection and 18S rRNA gene sequences of Cytauxzoon species in Iberian lynx (Lynx pardinus) in Spain. Parasitology. 2007;134:995-1001.

18. Millán J, Candela MG, Palomares F, Cubero MJ, Rodríguez A, Barral M, et al. Disease threats to the endangered Iberian lynx (Lynx pardinus). Vet. J. 2009;182:114-24. 
19. Gallusová M, Jirsová D, Mihalca AD, Gherman CM, D'Amico G, Qablan MA, et al. Cytauxzoon infections in wild felids from Carpathian-Danubian-Pontic space: further evidence for a different Cytauxzoon species in European felids. Parasitol. 2016;102:377-80.

20. Veronesi F, Ravagnan S, Cerquetella M, Carli E, Olivieri E, Santoro A, et al. First detection of Cytauxzoon infection in European wildcats (Felis silvestris silvestris) of Italy. Ticks Tick Borne Dis. 2016;7:853-8.

21. Hodžić A, Alić A, Duscher GG. High diversity of blood-associated parasites and bacteria in European wild cats in Bosnia and Herzegovina: a molecular study. Ticks Tick Borne Dis. 2018;9:589-93.

22. Ernst R, Ogeer J, McCrann D, Cross J, Strong-Townsend M, Friis H, et al. Comparative performance of IDEXX SDMA Test and the DLD SDMA ELISA for the measurement of SDMA in canine and feline serum. PLoS One. 2018;13:e0205030.

23. Carret C, Walas F, Carcy B, Grande N, Précigout É, Moubri K, et al. Babesia canis canis, Babesia canis vogeli, Babesia canis rossi: differentiation of the three subspecies by a restriction fragment length polymorphism analysis on amplified small subunit ribosomal RNA genes. Eukaryot. Microbiol. 1999;46:298-301.

24. Katargina O, Geller J, Vasilenko V, Kuznetsova T, Järvekülg L, Vene S, et al. Detection and characterization of Babesia species in Ixodes ticks in Estonia. Vector Borne Zoonotic Dis. 2011;11:923-8.

25. Hrazdilová K, Myśliwy I, Hildebrand J, Buńkowska-Gawlik K, Janaczyk B, Perec-Matysiak A, et al. Paralogs vs. genotypes? Variability of Babesia canis assessed by $18 \mathrm{~S}$ rDNA and two mitochondrial markers. Vet. Parasitol. 2019;266:103-10.

26. Jakob W, Wesemeier HH. A fatal infection in a Bengal tiger resembling cytauxzoonosis in domestic cats. Comp. Pathol. 1996;114:439-44.

27. Camacho-García AT. Piroplasma infection in dogs in northern Spain. Parasitol. 2006;138: 97-102.

28. Solano-Gallego L, Sainz Á, Roura X, Estrada-Peña A, Miró G. A review of canine babesiosis: the European perspective. Parasit. Vectors. 2016;9:336.

29. Reinacher M, Frese K. Untersuchungen zur Glomerulonephritis bei Hund und Katze. Tierarztl Prax. 1991;19:175-80.

30. Poli A, Falcone ML, Bigalli L, Massi C, Hofmann-Lehmann R, Lombardi S, et al. Circulating immune complexes and analysis of renal immune deposits in feline immunodeficiency virus-infected cats. Exp. Immunol. 2012;101:254-8.

31. Hosie MJ, Addie D, Belák S, Boucraut-Baralon C, Egberink H, Frymus T, et al. Feline immunodeficiency. ABCD guidelines on prevention and management. Feline Med. Surg. 2009;11:77584.

32. Harley L, Langston C. Proteinuria in dogs and cats. Vet. J. 2012;53:631-8.

33. Relford R, Robertson J, Clements C. Symmetric dimethylarginine: improving the diagnosis and staging of chronic kidney disease in small animals. Vet Clin Small Anim. 2016;46:941-60. 
34. Cohn LA, Birkenheuer AJ, Brunker JD, Ratcliff ER, Craig AW. Efficacy of atovaquone and azithromycin or imidocarb dipropionate in cats with acute cytauxzoonosis. Vet. Intern. Med. 2011;25:55-60.

35. Kier AB, Wagner JE, Morehouse LG. Experimental transmission of Cytauxzoon felis from bobcats (Lynx rufus) to domestic cats (Felis domesticus). J. Vet. Res. 1982;43:97-101.

36. Blouin EF, Kocan AA, Glenn BL, Kocan KM, Hair JA. Transmission of Cytauxzoon felis Kier, 1979 from bobcats, Felis rufus (Schreber), to domestic cats by Dermacentor variabilis (Say). Wildl. Dis. 1984;20:241-3.

37. Reichard MV, Meinkoth JH, Edwards AC, Snider TA, Kocan KM, Blouin EF, et al. Transmission of Cytauxzoon felis to a domestic cat by Amblyomma americanum. Vet. Parasitol. 2009;161:110-5.

38. Balzer S, Mölich T, Streif S, Tiesmeyer A, Thein J, Nowak C. Status der Wildkatze in Deutschland. Nat. Landsch. 2018;93:146-52.

39. Steyer K, Kraus RH, Mölich T, Anders O, Cocchiararo B, Frosch C, et al. Large-scale genetic census of an elusive carnivore, the European wildcat (Felis silvestris). Conserv. Genet. 2016;17:1183-99.

40. Petney TN, Pfäffle MP, Skuballa JD. An annotated checklist of the ticks (Acari: Ixodida) of Germany. Syst Appl Acarol. 2012;17:115-71.

\section{Figures}




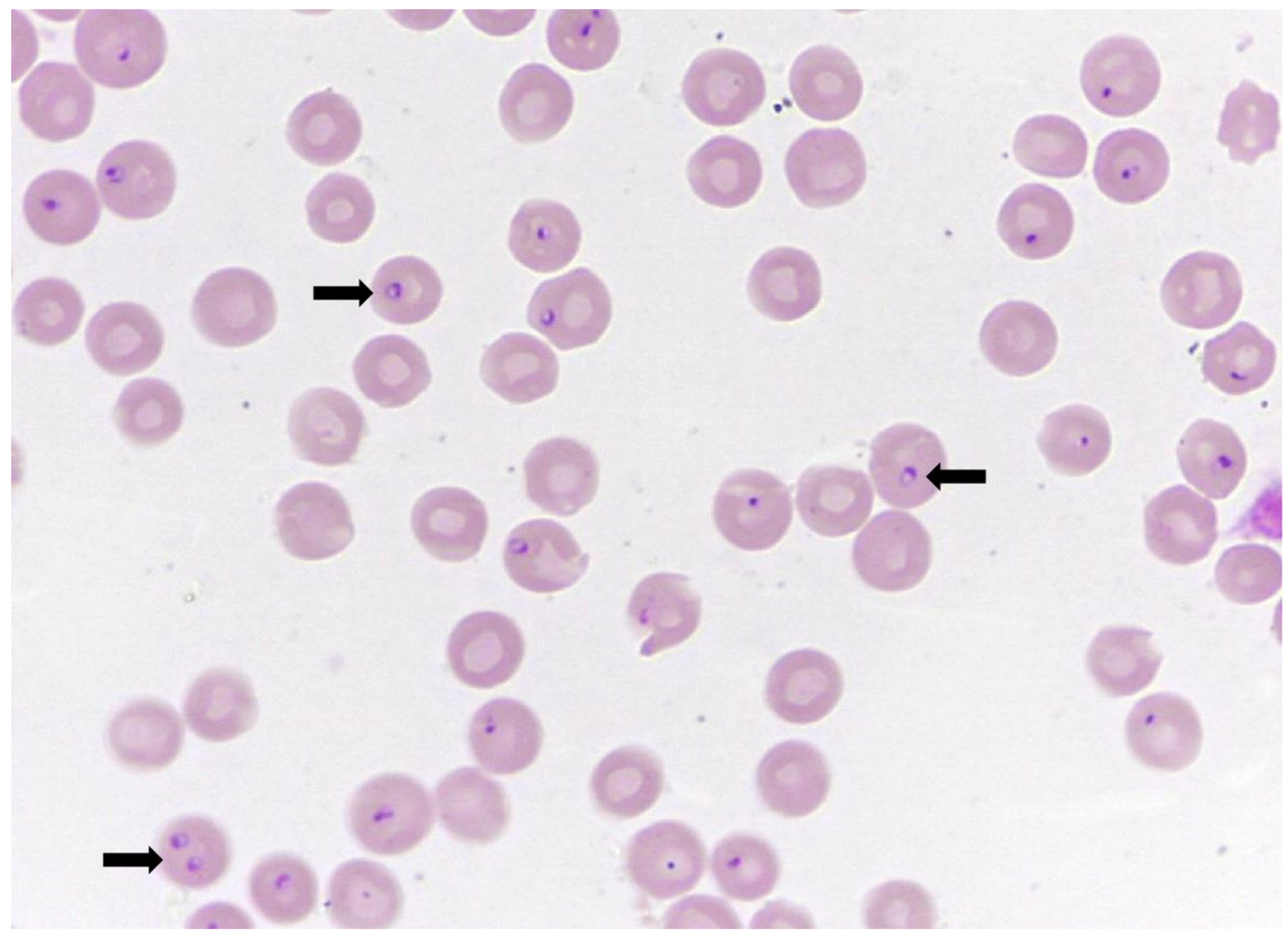

Figure 1

May Grünwald-Giemsa-stained peripheral blood smear (1000x). Cytauxzoon sp. appears as single or paired signet ring-shaped organisms (arrows) within erythrocytes. A high grade of parasitaemia can be observed. 